\title{
Management of adipsia by a behavioural modification technique
}

\author{
Stephen Johnston, Jayne Burgess, Tom McMillan, Richard Greenwood
}

\begin{abstract}
Adipsia combined with diabetes insipidus after hypothalamic damage may produce major difficulties in clinical management. If there is an associated memory impairment it may be impossible to teach self-regulation of fluid balance, necessitating long-term hospital supervision. The successful use of a behaviour modification technique to achieve independent drinking and allow discharge from hospital into the community is described in a patient with adipsia, diabetes insipidus and memory impairment resulting from the removal of a craniopharynigioma.
\end{abstract}

Deficient thirst sensation is known to occur after damage to osmoreceptors in the anterior hypothalmus. ${ }^{1}$ In the presence of a high plasma osmolality there is an inappropriate lack of thirst and water losses are not replenished by drinking. In addition, damage to adjacent supraoptic nuclei may cause defects in antidiuretic hormone (ADH) secretion, with hormone secretion refractory to either hyper or hypo-osmolar stimuii. Such patients with combined adipsia and diabetes insipidus lack internal defence mechanisms against both under and over hydration. Their clinical management can be extremely difficult, and the patients must be taught to adjust their daily fluid intake according to some objective measure of their state of hydration (for example, body weight). When the absence of thirst sensation together with a lack of $\mathrm{ADH}$ secretion is associated with poor concentration and memory impairment, selfmodulation of fluid intake becomes unreliable. We report a patient in whom a behaviour modification technique resulted in self-control of fluid balance. This had profound implications for long term medical care and independence in everyday living.

Regional Neurological Rehabilitation Unit, Homerton Hospital, London, UK

$S$ Johnston

J Burgess

T McMillan

R Greenwood

Correspondence to: Dr Greenwood,

Regional Neurological

Rehabilitation Unit

Rehabilitation Unit,

Lomdon E9 6SR, UK.

Received 12 April 1990 and in revised form 14 June 1990. Accepted 12 July 1990 weeks, it became evident that both her thirst sensation and her memory were impaired. She became water intoxicated with a plasma sodium concentration of $110 \mathrm{mmol} / \mathrm{l}$ (range 135-146). Subsequent control of her fluid balance proved difficult with swings in plasma sodium concentrations from 110 to $167 \mathrm{mmol} / \mathrm{l}$. Stability was eventually achieved on an acute medical ward with a regime of subcutaneous DDAVP and strict fluid balance. She remained reluctant to drink and, without constant supervision, easily became dehydrated: subsequent adjustments in her fluid intake resulted in violent swings in her plasma osmolality (fig, phase 0 ). In addition to her lack of thirst sensation, it was clear that the post-operative cognitive defect resulted in a lack of voluntary initiative to start or complete a drink and an inability to selfmonitor daily fluid intake. It was felt that without constant hospital supervision on an acute medical ward, there was risk of death from either dehydration or water intoxication.

On the basis of a reading test ${ }^{2}$ premorbid IQ was estimated to be 102 . Eight months postoperatively, verbal IQ was 77 and performance IQ $60 .{ }^{3}$ Eighteen months after surgery, verbal IQ had increased to 89 , but remained significantly lower than expected.

Memory was severely impaired. On the Wechsler Memory Scale (Revised) attention/ concentration and delayed memory were two standard deviations below average some fifteen months post injury. She scored poorly on the Rivermead Behavioural Memory Test ${ }^{4}$ seven months post-surgery $(31 / 76)$. Although her score had improved to some extent three months later (49/76), her memory remained impaired and had not improved overall when retested eighteen months post-operatively (49/ 76). Little further change was observed on this test some twelve months later (54/76).

She was transferred five months postoperatively to the rehabilitation unit with the aim of using a behaviour modification technique to achieve independent drinking, using an individualised token economy programme. The first goal was to achieve a reliable daily intake of the required volume of fluid, as determined by daily weight and serum osmolality levels (fig, phase 1 and 2). The second goal was to enable the patient to initiate drinking in a reliable way without the need for constant supervision (phase 3 and 4). In phase 1 (beginning nine months post-operatively), the patient was prompted by staff to initiate drinking, her daily fluid requirement being divided 


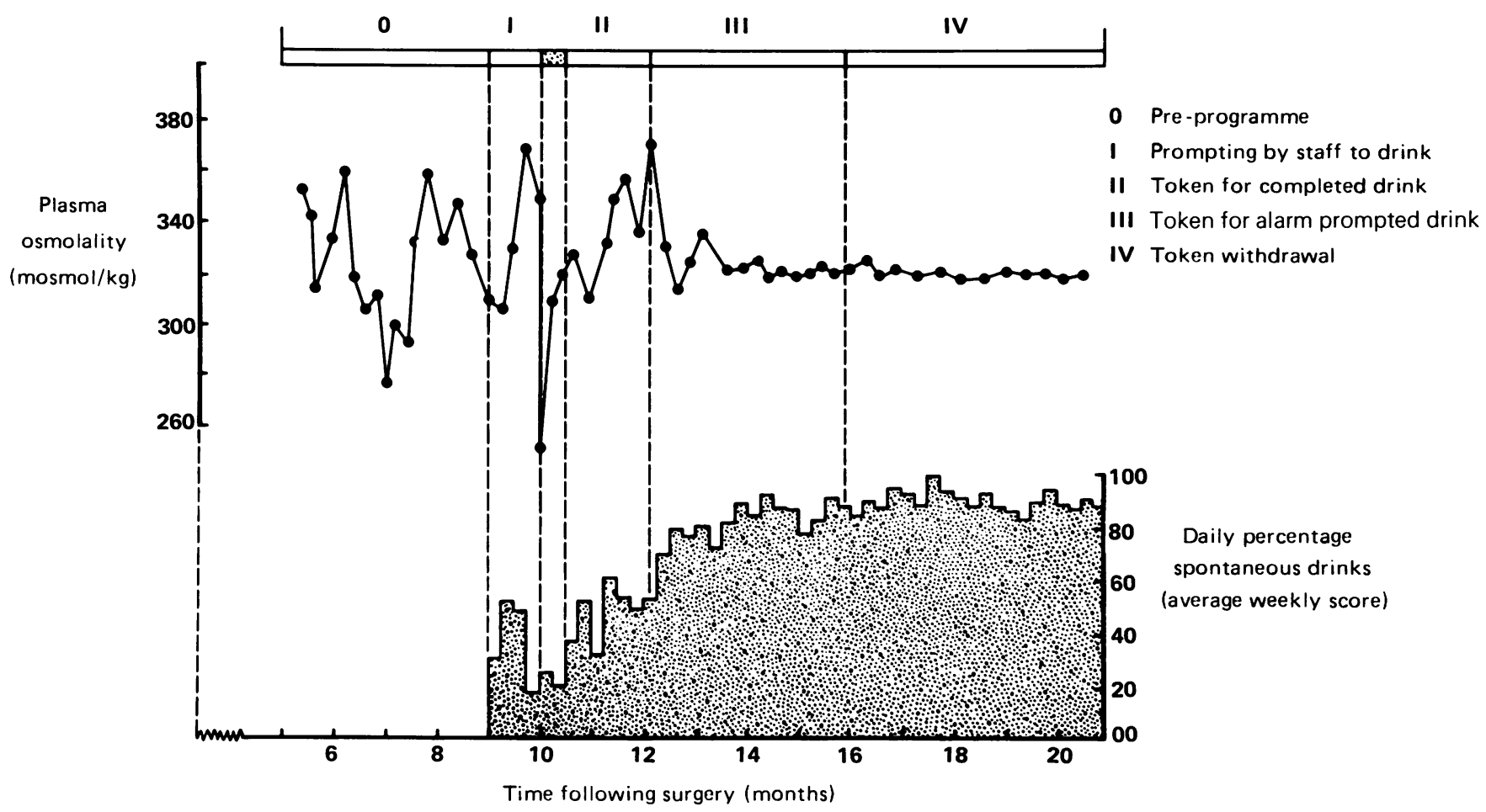

Figure The stabilisation of plasma osmolality with the introduction of the behaviour programme and the achievement of drinking prompted by a wristwatch alarm. Phase 0: failure to achieve stable osmolalities pre-programme. Phase 1: staff prompting initiation and completion of drinking with hypo-osmolar failure. Phase 2: alarm or staff prompting initiation of drinking, completion being rewarded by a token. Phase 3: removal of staff prompts, tokens continue. Phase 4: tokens stopped, alarm prompts continue with charting.

into ten equal drinks. She also needed considerable prompting to finish each drink because of poor concentration and memory. It became evident that it was not possible for the programme to be carried out consistently by all staff and three weeks into the programme she became hypo-osmolar and lost consciousness (fig), necessitating revision of the programme and consistency of staff input.

In phase 2 (10 months post-operatively), the required total daily fluid volume, which at that time varied but averaged two litres, was placed in a jug, or later in a vacuum flask, and the patient was provided with a cup with a level marked at one tenth of the day's required intake. The cup could be filled to the level with cold water from the flask or exchanged for any other drink at each of the specified drinking times during the day. She was prompted to drink ten times a day, using a wristwatch alarm; and a daily record was kept of the number of drinks taken, whether prompted by the alarm, or by the therapy staff. If the required amount of fluid was consumed at a drinking time, this was deemed a successful drink, and the patient received a card token as reinforcement. The tokens were collected by the patient and at the end of the day could be exchanged for a reward chosen from a "menu" devised by the patient and staff. For example, ten tokens could be exchanged for a magazine, fifteen for handcream, and fifty for a trip outside the Unit with therapy staff. The "cost" of these rewards were such that especially reinforcing rewards required reasonable improvement of baseline levels of drinking. After three weeks of phase 2, nine out of ten daily drinks were completed successfully, with an average drink rate in response to the alarm of $50 \%$ to $60 \%$.

In phase 3 (12 months post-operatively), tokens were only given for drinking in response to the alarm and within a further month this rate had increased from $80 \%$ to $90 \%$ with consistently stable blood osmolalities (fig). The patient felt well and consistently high drink rates were achieved in the Unit and at home at weekends, when the programme was monitored by her mother.

In phase 4 (16 months post-operatively), tokens were withdrawn and verbal reinforcement was given together with visual feedback of fluid intake by the use of a chart that the patient completed each day. Drink rates continued at $80 \%$ to $90 \%$ in response to the alarm, she began to attend a day centre and spent increasing amounts of time at home before discharge 18 months after her surgery.

At follow up a year after discharge, she continued to remain successfully established in the community. She charted her daily drink rates, and was still prompted by the wristwatch alarm to monitor her drinking. Her plasma osmolality stabilised at about $320 \mathrm{mosmol} / \mathrm{kg}$ and she remained adipsic.

\section{Discussion}

Osmoreceptors in the anterior hypothalmus control both the thirst centre and the rate of ADH secretion from the posterior pituitary. ${ }^{6}$ Damage to the hypothalamus or pituitary may impair ADH secretion, causing cranial diabetes insipidus, but the rise in plasma osmolality is matched by intense thirst sensation. However, 
the close proximity in the hypothalamus of the ' osmoreceptors controlling thirst and the neurons of the supraoptic nuclei secreting $\mathrm{ADH}$ means that damage to both centres may occur together. ${ }^{7}$ With no desire to drink, patients must learn to regulate their own fluid intake according to their state of hydration, using a sliding scale of fluid intake related to body weight. This was made impossible in our case by a third factor, cognitive impairment with loss of memory and concentration. In this context, independence seemed an impossible goal until the patient was trained to drink again using a behaviour modification technique.

Behavioural programmes with suitable "token" reinforcements have been successfully used to treat a variety of disorders of behaviour and function resulting from brain injury..$^{89}$ Tokens were used in this case to reinforce required drinking behaviour. Their introduction during Phase II and III improved the alarm prompted drink rate, and the figure shows that the serum osmolality stabilised as control of drinking improved. Several important points must be remembered if such a programme is to be successful.

Firstly, the rewards for which the tokens are exchanged must be appropriate to the individual patient. This is particularly important in brain damaged patients in whom apparently rewarding social activities may be totally meaningless if the patient is unable to appreciate their significance. Initial observation of patient behaviour must thus identify rewards specific to the individual. ${ }^{10}$ Secondly, tokens must be given as soon as possible after the completed behaviour for the behaviour to be reinforced; this is especially so in patients with memory disorders. Thirdly, the programme must be maintained consistently by all staff involved in the patient's care and reinforcement only given when the desired goal is achieved. The lack of consistency in the supervision of this programme led to its breakdown in Phase I. Only meticulous attention to daily routine allowed the tokens to become a significant reinforcement to the patient, initially allowing drinks to be completed successfully, and then prompted by her alarm, so that independent drinking was achieved. These requirements, and particularly the consistency of implementation, make the programme a difficult if not impossible undertaking in an acute medical ward.

Although stability in serum osmolality was achieved with behaviour modification, the final osmolality remained chronically elevated at about $320 \mathrm{mosmol} / \mathrm{kg}$ with an associated serum sodium of about $155 \mathrm{mmol} / \mathrm{l}$. There was no clinical evidence of dehydration and the patient functioned well at these levels. Chronic hyperosmolar states are recognised in association with adipsia, and in some instances may be related to selective resetting of the osmostat. ${ }^{7}$

It is significant that self monitoring of drinking was learned with no objective change in memory or cognitive function. This suggests that the development of independent drinking occurred as a direct result of behaviour modification, and not due to natural recovery of memory or cognitive function. This case demonstrates how such a technique can be used in cognitively impaired patients to achieve regular drinking and subsequent independence for the patient.

1 Anderson B, McCann SM. The effects of hypothalamic lesions on the water intake of the dog. Acta Physiol Scand 1956;35:312-20.

2 Nelson H. The National Adult Reading Test. Windsor: NFER-Nelson, 1982

3 Weschler D. Weschler Adult Intelligence Scale. New York: The Psychological Corporation, 1955.

4 Wilson B, Cockburn J. The Rivermead Behavioural Memory Test. Fareham: Thames Valley Test Company, 1982.

5 Ayllon Azrin. The token economy: a motivational system for therapy and rehabilitation. New York: Appleton, 1968.
then

therapy and rehabilitation. New York: Appleton, 1968.
6 Fitzsimons JT. Thirst. Physiol Rev 1972;52:468-561.

7 Robertson GL, Aycinena P, Zerbe RL. Neurogenic disorders of osmoregulation. Am J Med 1982;72:339-53.

8 Eames $\mathrm{P}$, Wood R. Rehabilitation after severe brain injury: a follow up study of a Behaviour Modification Approach. J Neurol Neurosurg Psychiatry. 1985:48(7):513-619.

9 Wood R. Brain injury rehabilitation: a neurobehavioural approach. Beckenham: Croom Helm, 1987.

10 Mikula JA. Non-pharmacological management in brain injury rehabilitation. In: Miner ME, Wagner KA, eds. Neurotrauma - treatment, rehabilitation and related issues, Vol 2. London: Butterworths, 1987. 